$T^{*} W^{*}$. If, then, $T X \neq W$, there is a sequence $w_{n}^{*}$ in $W^{*}$ such that $\left\|w_{n}^{*}\right\| \rightarrow 1$, while $T^{*} w w_{n}^{*} \rightarrow 0$. $T^{*}$ being $1-1$ on $W^{*}$, it cannot be an open mapping on to $T^{*} W^{*}$, whence the last subspace is not closed.

\title{
REFERENCES
}

1. S. Banach, Théorie des operations linéaires, Monografie Matematyczne, Warsaw, 1932; pp. 145-152.

2. N. Dunford and J. Schwartz, Linear operators. I, Interscience, New York, 1958; Lemma 3, p. 488.

UNIVERSITY OF ILLINOIS

\section{A SHORT PROOF OF JACOBI'S FOUR SQUARE THEOREM}

\section{CARLITZ ${ }^{1}$}

Let $R_{4}(n)$ denote the number of representations of $n$ as a sum of four squares and let $R_{4}^{\prime}(4 m)$, where $m$ is odd, denote the number of representations of $4 m$ as a sum of four odd squares. It is familiar that

$$
R_{4}^{\prime}(4 m)=16 \sigma(m)
$$

and

$$
R_{4}(n)=\left\{\begin{aligned}
8 \sigma^{\prime}(n) & (n \text { odd }) \\
24 \sigma^{\prime}(n) & (n \text { even })
\end{aligned}\right.
$$

where

$$
\sigma(n)=\sum_{d \mid n} d, \quad \sigma^{\prime}(n)=\sum_{d \mid n ; d \text { odd }} d .
$$

These results can be proved rapidly as follows. In the usual notation of elliptic functions put [2, Chapter 21]

$$
\lambda=k^{2}=\frac{\theta_{2}^{4}}{\theta_{3}^{4}}, \quad 1-\lambda=\frac{\theta_{0}^{4}}{\theta^{4}} .
$$

Then

Received by the editors May 21, 1965.

1 Supported in part by NSF grant GP-1593. 


$$
1-\lambda=\prod_{n=1}^{\infty}\left(\frac{1-q^{2 n-1}}{1+q^{2 n-1}}\right)^{8} .
$$

Now, it follows easily from $q=\exp \left[-\pi K^{\prime} / K\right]$ that $[2$, p. 521]

$$
\begin{aligned}
\frac{1}{q} \frac{d q}{d \lambda} & =-\frac{\pi}{K^{2}}\left(K \frac{d K^{\prime}}{d \lambda}-K^{\prime} \frac{d K}{d \lambda}\right) \\
& =\frac{1}{\lambda(1-\lambda) \theta_{3}^{4}} .
\end{aligned}
$$

Thus logarithmic differentiation of (3) yields

$$
\theta_{2}^{4}=\lambda \theta_{3}^{4}=16 \sum_{1}^{\infty} \frac{(2 n-1) q^{2 n-1}}{1-q^{2(2 n-1)}}=16 \sum_{m=1 ; m \text { odd }}^{\infty} \sigma(m) q^{m}
$$

and (1) follows at once.

Similarly from

$$
\lambda=2 q \prod_{1}^{\infty}\left(\frac{1+q^{2 n}}{1+q^{2 n-1}}\right)^{8}
$$

we get

$$
\theta_{0}^{4}=(1-\lambda) \theta_{3}^{4}=1+8 \sum_{1}^{\infty}\left(\frac{2 n q^{2 n}}{1+q^{2 n}}-\frac{(2 n-1) q^{2 n-1}}{1+q^{2 n-1}}\right) .
$$

Replacing $q$ by $-q$ this becomes

$$
\begin{aligned}
\theta_{3}^{4} & =1+8 \sum_{1}^{\infty}\left(\frac{2 n q^{2 n}}{1+q^{2 n}}+\frac{(2 n-1) q^{2 n-1}}{1-q^{2 n-1}}\right) \\
& =1+16 \sum_{n=1}^{\infty} \sigma^{\prime}(n) q^{2 n}+8 \sum_{n=1}^{\infty} \sigma^{\prime}(n) q(n)
\end{aligned}
$$

and (2) follows at once.

For the standard elliptic function proof of (2) see for example [1, pp. 205-206].

\section{REFERENCES}

1. A. Hurwitz and R. Courant, Funktionentheorie, Springer, Berlin, 1929.

2. E. T. Whittaker and G. N. Watson, Modern analysis, 4th ed., Cambridge Univ. Press, New York, 1927.

Duke University 